\title{
Postoperative Analgesia in Thoracic Surgery: A Comparison between Continuous Paravertebral Nerve Block and Continuous Incisional Infusion with OnQ Pain Relief System
}

\author{
Germano De Cosmo ${ }^{1 *}$, Bruno Federico ${ }^{2}$, Flaminio Sessa ${ }^{3}$, Federico Fiorini ${ }^{4}$, Giusy Fortunato ${ }^{5}$ and Elisabetta Congedo ${ }^{6}$ \\ ${ }^{1}$ Associate Professor, Department of Anesthesiology and Intensive Care, Policlinico A. Gemelli, Largo A. Gemelli 8, 00168 Rome, Italy \\ ${ }^{2}$ Department of Anesthesiology and Intensive Care, Catholic University of the Sacred Heart, Rome, Italy \\ ${ }_{3,4,5,6}$ Department of Health and Sport Sciences, University of Cassino, Italy
}

\begin{abstract}
Objectives: Thoracotomy is one of the most painful surgical stimuli and inadequate management of postthoracotomy pain is often associated with pulmonary and cardiac complications. The aim of this prospective, randomized, double-blinded study was to compare continuous paravertebral block versus continuous incisional infusion with OnQ Pain Relief System.
\end{abstract}

Methods: Forty eight patients, undergoing thoracotomy for elective lobectomy, were randomized to receive a continuous paravertebral infusion of bupivacaine $0.25 \%$ through an elastomeric pump which delivers $0.1 \mathrm{~mL} / \mathrm{Kg} / \mathrm{h}$ (group A) or a continuous incisional infusion of bupivacaine $0.25 \%$ at an infusion rate of $4 \mathrm{~mL} / \mathrm{h}$ with OnQ Pain Relief System (group B). Both infusions were started before wound closure, and continued for 48 postoperative hours. General anaesthesia was standardized. In the recovery room, patients were provided with intravenous morphine patient-controlled analgesia (PCA). Visual analogue scale at rest (VASr) and when coughing (VASi), rescue patientcontrolled analgesia morphine consumption, hemodynamic, time to ambulation and side-effects were evaluated within $48 \mathrm{~h}$.

Results: The two groups were comparable regarding to patients' number and characteristics, type of surgery, time to ambulation and side-effects; postoperative hemodynamic profile was stable in all the patients. Absolute pain scores were low in both groups; patients in group A reported significant lower VASr and VASi values during the postoperative 48 hours compared with group $B(p<0.001)$. Total morphine consumption and PCA requests number were significantly lower in group $A$ than in group $B(p=0.05$ and $p<0.01)$.

Conclusions: Continuous incisional infusion of local anesthetic is not as effective as paravertebral analgesia after thoracotomy.

Keywords: Paravertebral block; Thoracotomy pain; Wound infiltration; ON-Q pain relief system

\section{Introduction}

Thoracotomy is one of the most painful surgical stimuli currently practiced. Inadequate management of post-thoracotomy pain can increase patient's discomfort and it is often associated with postoperative pulmonary and cardiac complications [1,2]. Because the difficulty in pain control, many local and regional analgesic approaches have been suggested in order to reduce the side effects associated to intra and postoperative systemic opioid administration [3]. For many years thoracic epidural analgesia was considered to be the most effective method of pain control and it remains the gold standard to treat post-thoracotomy pain; however, in the last few years evidences have suggested that paravertebral nerve block can be at least as effective as epidural analgesia for analgesia in thoracotomy; moreover, it is associated with fewer side effects (less hypotension, less nausea and vomiting, less pulmonary complications, reduced incidence of urinary retention) [4-6]. Despite its utility in reducing pain, thoracic paravertebral block is not free from risks such as pneumothorax, pleural puncture, and intravascular injection [5]. Recently incisional administration of local anesthetics has been shown to have a role in the management of postoperative pain in several type of surgery. To reduce the unpleasant effect of the brief analgesic duration of most local anesthetics, new devices have been developed that deliver a continuous infusion of analgesics to surgical site. The aim of this study was to compare two analgesia techniques for post-thoracotomy pain: continuous paravertebral block versus continuous incisional infusion; the main outcome variables assessed will be rescue total amount of morphine administered, number of patient controlled analgesia (PCA) requests, postoperative pain and the time required for the patient's deambulation [7]

\section{Materials and Methods}

After approval by the Ethics Committee of the Catholic University of the Sacred Heart of Rome and subsequent collection of patient's written informed consent, 48 patients, aged between 50-70 years, ASA physical status class II-III, Body Mass Index (BMI) between 18.5-29.9, undergoing postero-lateral thoracotomy for lobectomy for stage I or II non small cell lung cancer, were enrolled. The exclusion criteria were: lack of patient's consent, failure of the patients to understand

*Corresponding author: Germano De Cosmo, Associate Professor Department of Anesthesiology and Intensive Care, Policlinico A. Gemelli, Largo A. Gemelli 8, 00168 Rome, Italy, Tel: +390630154507; Fax: +39063013450; E-mail: gdecosmo@rm.unicatt.it

Received November 28, 2011; Accepted December 30, 2011; Published January 17,2012

Citation: De Cosmo G, Federico B, Sessa F, Fiorini F, Fortunato G, et al. (2012) Postoperative Analgesia in Thoracic Surgery: A Comparison between Continuous Paravertebral Nerve Block and Continuous Incisional Infusion with OnQ Pain Relief System. J Anesthe Clinic Res 4:279. doi:10.4172/2155-6148.1000279

Copyright: (C) 2012 De Cosmo G, et al. This is an open-access article distributed under the terms of the Creative Commons Attribution License, which permits unrestricted use, distribution, and reproduction in any medium, provided the original author and source are credited. 

between Continuous Paravertebral Nerve Block and Continuous Incisional Infusion with OnQ Pain Relief System. J Anesthe Clinic Res 4:279. doi:10.4172/2155-6148.1000279

or cooperate with visual analogue pain score or patient controlled analgesia (PCA) use for rescue analgesia, coagulopathy, allergy to amide-type local anesthetics or opioids, patients with chronic pain or receiving preoperative analgesics. All the patients were informed that giving informed consent to participate in the study did not exclude the possibility to withdraw at any time. The day before surgery, during pre-operative visit and enrollment to the study, patients were instructed about Visual Analogue Scale (VAS) for pain assessment by an anesthesiologist involved in the study and employed to collect data. VAS is a horizontal line, $10 \mathrm{~cm}$ in length, ranging from $0 \mathrm{~cm}$ (no pain at all) to $10 \mathrm{~cm}$ (worst imaginable pain). The patients mark on the line the point they feel corresponds to their pain.

Moreover, patients were familiarized about the use of PCA device to alleviate postoperative pain. Before surgery, patients were assigned, using random allocation software to support a blocked randomization, to two groups: A and B. In group A, postoperative analgesia was assured by continuous paravertebral nerve block (PVB), while in group B by postoperative continuous incisional infusion of $0.25 \%$ bupivacaine via the ON-Q Soaker 20-gauge catheters connected to an elastomeric balloon pump (OnQ Pain Buster, I-Flow Corporation, CA). The day of surgery, patients were premedicated with diazepam 8-10 mg given orally $30 \mathrm{~min}$ before induction of anesthesia. Intra-operative monitoring included heart rate, pulse oximetry, five-lead electrocardiogram, and end-tidal carbon dioxide. Arterial blood pressure was measured upon arrival of the patients in the operating theatre (baseline value) and continuously, by means of radial artery catheter, until discharge from the recovery room. In both groups general anesthesia was induced with propofol $2 \mathrm{mg} / \mathrm{Kg}$ and fentanyl $2 \mathrm{mcg} / \mathrm{Kg}$. The placement of an endobronchial double-lumen was facilitated with cis-atracurium 0.15 $\mathrm{mg} / \mathrm{Kg}$. Maintenance of anesthesia was provided by using sevoflurane and fentanyl according to clinical necessity. Total consumption of fentanyl was recorded at the end of surgery. Therefore, after the induction of the anesthesia and before starting the surgery, all the patients were turned in the lateral position, with the side to be blocked and operated on upward, and a PVB was performed to them at thoracic level, from T4 to T6, with an 18-gauge Tuohy needle, using loss-ofresistance technique according to Eason and Wyatt [8]. After the spinous processes associated with the nerves to be blocked have been identified, a 25 gauge needle was inserted $2.5 \mathrm{~cm}$ lateral from the cephalad edge of the thoracic vertebral spinal processes and a skin wheal of $1 \%$ lidocaine was placed over the transverse processes from T4 to T6. Even if patients were under general anesthesia and fentanyl was already given, however we performed a skin wheal of $1 \%$ lidocaine to mark injection sites after that with a small 25 gauge needle we have identified the transverse processes. Afterwards, an 18-gauge Tuohy needle was inserted until the transverse processed have been contacted; once the transverse process was contacted, the needle was partially withdrawn and redirected caudally and advanced approximately $1.5-2 \mathrm{~cm}$ deeper until a subtle loss of resistance was felt by the anesthesiologist performing PVB. Loss of resistance indicates entry into the paravertebral space.

After a negative aspiration test, $5 \mathrm{~mL}$ of bupivacaine $0.5 \%$ was injected to each site. A 20-gauge epidural catheter was then inserted through the 18-gauge Tuohy needle into the paravetebral space at T6 level only in patients of group A. At the end of surgery a continuous paravertebral infusion of bupivacaine $0.25 \%$, through an elastomeric pump set at a rate of $0.1 \mathrm{~mL} / \mathrm{Kg} / \mathrm{h}$, was started and continued for 48 hours in group A. The malpositioning of the paravertebral catheter could be checked by the surgeon during the surgery. In group $\mathrm{B}$, the ON-Q Soaker 20-gauge multiorifice catheters were placed in the thoracotomy area by the surgeon. One catheter was placed at the level of the surgical wound site near the fifth intercostals nerve bundle and the other one was placed above the fascia in the subcutaneous space. The catheters were connected to an elastomeric pump delivering bupivacaine $0.25 \%$ at a fixed infusion rate of $4 \mathrm{~mL} / \mathrm{h}$ for 48 hours. The rate of the elastomeric pumps was obscured by a patch in order to assure that anesthetist investigator collecting data and patient were blinded to the type of analgesic device used. Paravertebral infusion and incisional infiltration were started at the same time at the skin suture. In the recovery room, $90 \mathrm{~min}$ after extubation (when patient was fully awake), patient-controlled analgesia (PCA) was provided using an electronic device with morphine $0.6 \mathrm{mg} / \mathrm{ml}$, set to deliver an intravenous bolus of $1 \mathrm{ml}$ with a lockout interval of $10 \mathrm{~min}$ and a $7 \mathrm{mg}$ dose limit over 4 hours. If, before PCA beginning, patients needed of analgesia, they received $2 \mathrm{mg}$ boluses of morphine every $5 \mathrm{~min}$ until their VAS was lesser than 4 . Total amount of morphine that patient received and numbers of PCA requests were recorded. Arterial blood pressure (BP), heart rate (HR), respiratory rate and oxygen saturation were registered throughout the study period. Hypotension was defined as a $20 \%$ decrease of systolic BP compared with baseline and a systolic $\mathrm{BP}$ less than $90 \mathrm{mmHg}$. Bradycardia was defined as a heart rate less than 50 beats per minute, and bradypnoea as a respiratory rate less than 10 breaths per minute. Patients were assessed for pain at rest (VASr) and while coughing (VASi) hourly for the first postoperative 4 hours and subsequently every 4 hours for the next 44 hours. Moreover, time to ambulation and the occurrence of pruritus, nausea, vomiting and any adverse events were also recorded.

\section{Statistical Analysis}

Results were expressed as means \pm SD for quantitative variables and as percentage for categorical variables. The number of patients included in the study was based on a priori power analysis assuming $\alpha=0.05$ and $\beta=0.20$. A minimum of 22 patients for each group was required to achieve a power of $90 \%$ and to detect a minimum difference of $50 \%$ between the two groups in VAS score. Three different pain indicators were used: VAS (at rest and while coughing), total morphine consumption and number of PCA requests. The analysis was conducted on absolute values and on time-related measurements. For absolute values Mann-Whitney and Chi-squared test were used as appropriate. Analysis of variance (ANOVA) with intervals as repeated measures was used on time-related measurements. A value of $\mathrm{P}<0.05$ was considered as significant. Statistical analysis was performed with the use of Stata 11 .

\section{Results}

A total of 48 patients completed the study (24 for each group). No patients were excluded because of protocol deviation. The two groups were comparable regarding to patients' characteristics, type of surgery and time to ambulation; however, total morphine consumption was significantly lower in group A than in group B (Table 1). There were no complications during PVB or paravertebral space catheterisation. Intraoperative fentanyl consumption was similar in both groups (Group A: $265 \mathrm{mcg} \pm 55$ vs Group B: $261 \mathrm{mcg} \pm 48, \mathrm{p}=0.3$ ). No adverse events were seen during surgery and postoperatively. Values of systolic and diastolic arterial pressure and HR were stable in all patients during the study period. There was no statistically significant difference between the groups with regard to these variables. Bradypnoea or respiratory depression events were not observed in patients of both groups. As shown in Figures 1 and 2, both VASr and VASi increased after the first 4 hours post-operatively, achieving maximum pain peak at the $8^{\text {th }}$ hour; afterward, VASr and VASi decreased. Group A patients had significant 
Citation: De Cosmo G, Federico B, Sessa F, Fiorini F, Fortunato G, et al. (2012) Postoperative Analgesia in Thoracic Surgery: A Comparison between Continuous Paravertebral Nerve Block and Continuous Incisional Infusion with OnQ Pain Relief System. J Anesthe Clinic Res 4:279. doi:10.4172/2155-6148.1000279

Page 3 of 5

\begin{tabular}{|l|l|l|l|}
\hline & Paravertebral Block & On-Q & p-value** \\
\hline Age (years) & $59.2(8.3)$ & $61.3(10.1)$ & 0.21 \\
\hline \% Male & 54.2 & 58.3 & 0.77 \\
\hline BMI (kg/m2) & $24.4(2.2)$ & $25.1(2.5)$ & 0.29 \\
\hline \% Lob dx & 54.2 & 62.5 & 0.56 \\
\hline Duration of surgery; min & $108(39)$ & $110(42)$ & 0.3 \\
\hline Duration of anesthesia; min & $125(40)$ & $129(42)$ & 0.42 \\
\hline Morphine (mg) & $13.1(8.8)$ & $19.1(10.9)$ & 0.05 \\
\hline Time to walking (hours) & $27.3(11.1)$ & $31.5(12.2)$ & 0.16 \\
\hline
\end{tabular}

*Mean (Standard deviation) and percentage are reported for numerical and categorical variables, respectively ${ }^{* *}$ Chi-squared test was used for categorical variables, and Mann-Whitney test was used for numerical variables

Table 1: Patients characteristics and outcomes by group*

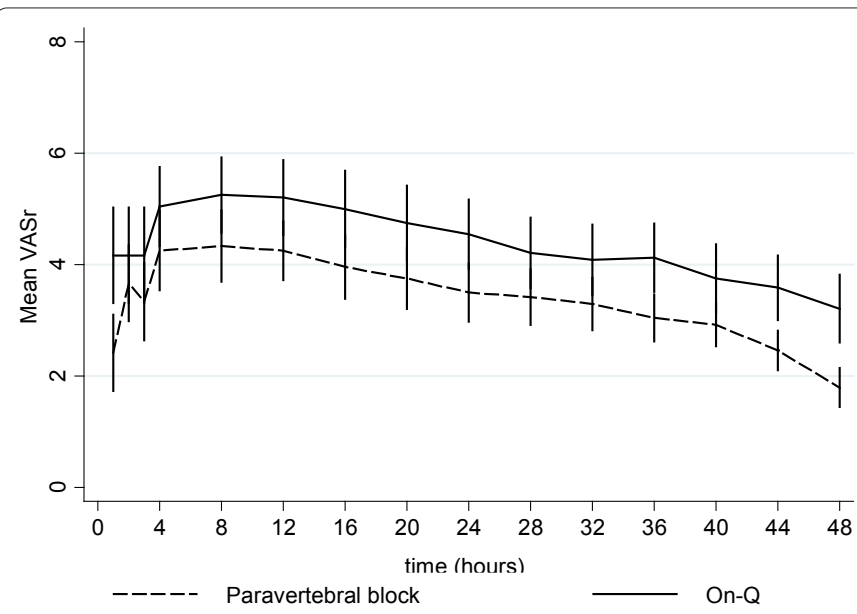

Figure 1: Mean VASr versus time (hours) after surgery by group Mean difference between On-Q and Paravertebral block: 0.99. Repeated measurements ANOVA - $p<0.001$ On-Q ----- Paravertebral block.

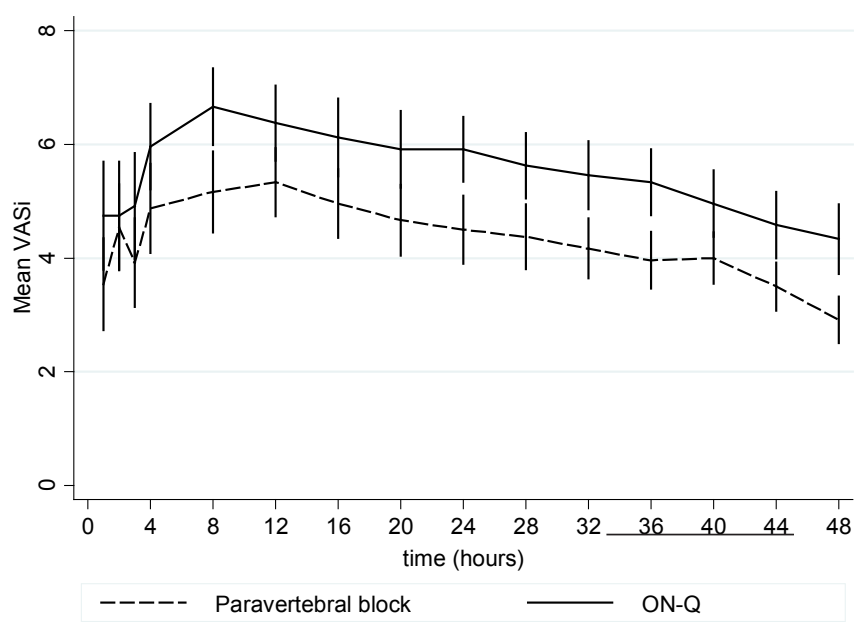

Figure 2: Mean VASi versus time (hours) after surgery by group Mean difference between On-Q and Paravertebral block: 1.15. Repeated measurements ANOVA - $p<0.001$ On-Q Paravertebral block.

lower VAS scores during the entire period of study $(\mathrm{p}<0.001)$. A peak in number of PCA requests was reached at 12 hours for both groups, decreasing in the following 36 hours; mean PCA requests was significant lower for group A compared to group B during the 48 postoperative hours $(\mathrm{p}<0.001)$.

\section{Discussion}

In this prospective randomized study we have utilized two types of analgesia technique in patients undergoing thoracothomy: continuous paraveretebral block and continuous incisional infusion with On-Q pain system. Patients belonging to group an experienced lower pain score than those to group $\mathrm{B}$. The rationale to perform this study was to demonstrate a valid alternative to manage post thoracotomy pain respect to the recognised effective analgesia technique: paravetrebral nerve block. Many different analgesia techniques are used for postoperative pain relief such as intravenous and intrathecal opioids, epidural analgesia, intercostals and paravertebral nerve blockade, interpleural infusion of local anaesthetics [3]. Efforts have been done to find the best method of pain relief with the minor side-effects.

In the last years it is increasing the use of devices that deliver local anesthetics continuously to the surgical site like the most recent On-Q painbuster system provided with two soaker catheters that infuse medication over a wider surgical area. This system has been investigated and approved for many type of surgery. There are few clinical studies assessing the effectiveness of On-Q pain system respect to conventional pain relief techniques in patients undergoing thoracotomy for lung surgery. For patients undergoing thoracotomy previous comparisons were made between On-Q pain relief system and thoracic epidural analgesia or intravenous administration of opioids showing decreased pain score and narcotic use with the former system $[9,10]$. This is the first study that compares the PVB block, a commom method of postthoracothomy analgesia, to the incisional infusion with local anesthetic by On-Q system. Thoracic epidural analgesia is considered the gold standard for post-thoracotomy pain. However, there are many reasons for which epidural technique cannot be performed and is contra-indicated. For example, the placement of the epidural catheter may be very difficult in patients who have undergone previous operation on the spine, in obese subjects or in patients with spine deformity. Moreover, epidural analgesia may be accompanied by complications such as hypotension, headache, nausea, urinary retention, pruritus and infections. There is evidence that thoracic paravertebral nerve block provide a good analgesia and has been shown to be as effective as epidural analgesia and to be accompanied by lower frequency of hypotension, lower postoperative nausea and vomiting, and better pulmonary function [46]. Moreover, PVB can be performed in those situations when epidural catheter cannot be inserted because it is contra-indicated. However, PVB realization is not completely free from risks being subject to the same types of complications associated with thoracic epidural analgesia: pneumothorax, central neuraxial block, hypotension, intravascular injection. Wound infiltration with a local anaesthetic was described for the first time in 1935 by Capelle [11]. Afterwards, Blades and Ford used this technique for gynaecological procedures reporting a decrease in opioid requirements [12]. The injection of a local anaesthetic at the surgical site reduces pain inhibiting transmission of the nociceptive impulses from the site of injury. Moreover, Hopf emphasized that the reduction in pain caused by local infiltration reduces the decreased wound perfusion and oxygenation induced by inflammatory response and increased catecholamines, thus improving wound healing $[13,14]$. A systematic review performed by Liu and colleagues confirmed the usefulness of this analgesic method during several painful surgical procedures, such as thoracic, cardiac, gynecologic or spinal surgery. Liu colleagues in their review emphasized that the success of this technique may be related to the type of surgery and to the level of tissue 

between Continuous Paravertebral Nerve Block and Continuous Incisional Infusion with OnQ Pain Relief System. J Anesthe Clinic Res 4:279. doi:10.4172/2155-6148.1000279

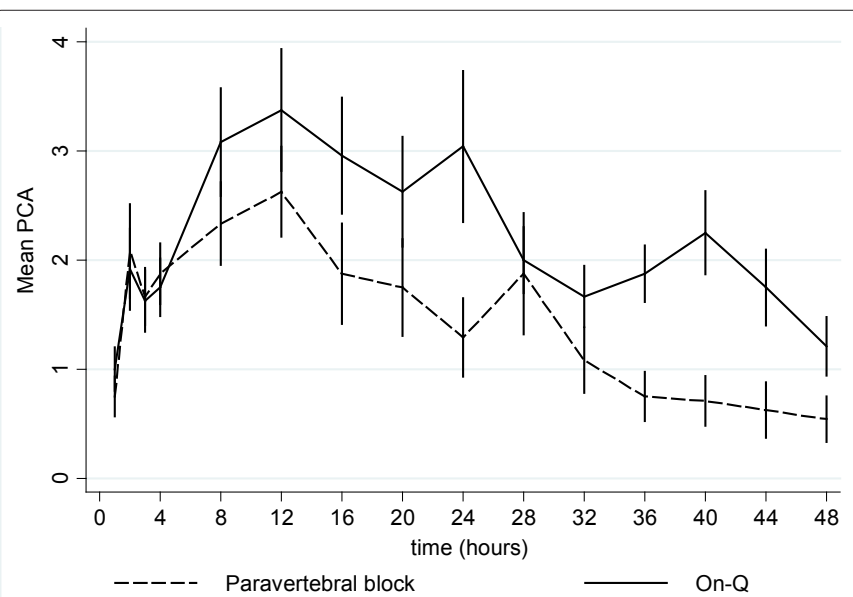

Figure 3: Mean of number of PCA requests every four hours versus time (hours) after surgery by group

Mean difference between On-Q and Paravertebral block: 0.69. Repeated measurements ANOVA - $p<0.001$

On-Q

Paravertebral block

where the infiltration takes place. In particular, if the catheter is placed subcutaneously there is no improvement of postoperative pain scores [15]. Forastiere and co-workers studied the effectiveness of continuous wound infusion of $0.5 \%$ ropivacaine by ON-Q system for postoperative pain after open nephrectomy showing that when this analgesic device is compared to placebo it decreases pain and narcotics [16]. Also Salvemini has reported a decreased narcotic use and lower pain score in patients who underwent main pulmonary resection due to lung neoplasm when incisional infiltration with ON-Q Pain Relief System is compared with traditional intravenous opioids [17]. Sidiropoulou and colleagues in their study on women undergoing modified radical mastectomy with axillary dissection compared preoperative PVB with $20 \mathrm{~mL}$ of ropivacaine $0.5 \%$ with continuous wound infiltration of ropivacaine $0.5 \%$ at a $2 \mathrm{~mL} / \mathrm{h}$ rate through ON-Q Pain Relief System placed subcutaneously and started at the end of surgery regarding to postoperative pain. They concluded that local anaesthetic infiltration by ON-Q Pain Relief System is comparable to single preoperative injection PVB for postoperative pain [18]. In our study we did not find evidence of a greater effectiveness of ON-Q device compared to PVB in patients who underwent thoracotomy. Furthermore, the greater efficacy of paravertebral infusion is already evident since the first hours after surgery. Inlike Sidiropoulou's study, we did not place the catheter only in the subcutaneous tissue but also near the intercostals nerve bundle expecting an adequate management of pain. The difference between our and Sidiropoulou's study may be due to the type of pain and to the time and way of PVB execution. Thoracotomy is considered to be one of the most painful surgical procedures for the extent of the surgical insult and it determines a high incidence of development of chronic pain. Moreover, in our study continuous incisional infiltration by ON-Q Pain System and continuous paravertebral infusion were started at the end of surgery before wound closure. Instead, Sidiropoulou performed a single injection of lidocaine $2 \%$ in the third thoracic paravertebral space in the preoperative period. Despite the fact that authors concluded that both techniques were effective, in the early postoperative period patients receiving PVB showed lower VAS scores compared to patients in the ON-Q group. Furthermore, in our study we did not use boluses but a continuous paravertebral infusion of local anesthetic and several studies comparing boluses with continuous infusion for the maintenance of PVB confirmed that continuous infusion is associated with an improvement of analgesia at all time points up to $48 \mathrm{~h}$ [19]. Furthermore, despite in patients of group A continuous PVB had been performed, their mobility was not affected.

A study performed by Wheatley and colleagues reported a reduction of patient's pain after thoracotomy when local surgical infiltration by ON-Q Pain Relief System was compared with a single-shot epidural analgesia in combination with $\mathrm{ON}-\mathrm{Q}$ and with single continuous epidural infusion [9]. In their retrospective study the authors used an ON-Q Pain Relief System with two catheters: one catheter was placed at the level of the pericostal sutures adjacent to the intercostals nerve bundle, and the other was placed above the fascia in the subcutaneous space. In our study we also used the ON-Q Soaker 20 Gauge catheters placed in the same position as those in Wheatley's study, but we did not obtain similar results. A possible explanation regarding to the different results could be that the authors administered a 5 to $10 \mathrm{~mL}$ bolus of $0.25 \%$ bupivacaine through the catheters just before incisional site was closed. Moreover, Wheatley's study has a retrospective design, therefore with associated limitations that reduce the power of its results.

The only study comparing thoracic paravertebral block with intercostals nerve block was that of Perttunen and colleagues [20]. In their study the authors compared three analgesia techniques for postthoracotomy pain: extradural, paravertebral and intercostals nerve block. The authors did not detect significant differences between the groups; however, unlike our study the authors blocked four intercostals nerves with a higher plasma concentration of local anesthetic in patients treated with intercostals nerve block.

In conclusion continuous incisional infusion of local anesthetics is not as effective as paravertebral analgesia to reduce pain after thoracotomy. Continuous PVB is associated with better pain relief without mobility worsening. However, we believe that in the future studies investigating a combination of these two methods without achieving toxic doses of local anesthetic should be performed to find the most effective, safety and easy to perform analgesia technique for the management of post thoracotomy pain.

\section{References}

1. Ballantyne JC, Carr DB, deFerranti S, Suarez T, Lau J, et al. (1998) The comparative effects of postoperative analgesic therapies on pulmonary outcome: cumulative meta-analyses of randomized, controlled trials. Anesth Analg 86: 598- 612.

2. Groban L, Dolinski SY, Zvara DA, Oaks T (2000) Thoracic epidural analgesia: its role in postthoracotomy atrial arrhythmias. J Cardiothorac Vasc Anesth 14 662-665.

3. De Cosmo G, Aceto P, Gualtieri E, Congedo E (2009) Analgesia in thoracic surgery: review.Minerva Anestesiol 75: 393- 400

4. Scarci M, Joshi A, Attia R (2010) In patients undergoing thoracic surgery is paravertebral block as effective as epidural analgesia for pain management? Interact Cardiovasc Thorac Surg 10: 92- 96.

5. Powell ES, Cook D, Pearce AC, Davies P, Bowler GM, et al. (2011) A prospective, multicentre, observational cohort study of analgesia and outcome after pneumonectomy. Br J Anaesth 106: 364- 370

6. Davies RG, Myles PS, Graham JM A (2006) comparison of the analgesic efficacy and side-effects of paravertebral vs epidural blockade for thoracotomya systematic review and metaanalysis of randomized trials. $\mathrm{Br} \mathrm{J}$ Anaesth 96 418- 426.

7. Naja Z, Lönnqvist PA (2001) Somatic paravertebral nerve blockade. Incidence of failed block and complications. Anaesthesia 56: 1184- 1188.

8. Eason MJ, Wyatt R (1979) Paravertebral thoracic block-a reappraisal. Anaesthesia 34: 638- 642 .

9. Wheatley GH, Rosenbaum DH, Paul MC, Dine AP, Wait MA, et al. (2005) 
Citation: De Cosmo G, Federico B, Sessa F, Fiorini F, Fortunato G, et al. (2012) Postoperative Analgesia in Thoracic Surgery: A Comparison between Continuous Paravertebral Nerve Block and Continuous Incisional Infusion with OnQ Pain Relief System. J Anesthe Clinic Res 4:279. doi:10.4172/2155-6148.1000279

Page 5 of 5

Improved pain management outcomes with continuous infusion of a local anesthetic after thoracotomy. J Thorac Cardiovasc Surg 130: 464- 468.

10. Rampersad SE, Rowell JC, Chang BM (2009) Comparison of the On-Q Painbuster Post-Op Pain Relief System to a thoracic epidural for control of postoperative thoracotomy pain in a child. Paediatr Anaesth 19: 1025- 1026

11. Capelle W (1935) Die Bedentung des Wundschmerzes und seiner ausschaltung fur danAblauf der Atmungbei Laparoto-mierten. Dtch Z Chi 246: 466.

12. Blades B, Ford WB (1950) A method of control of postoperative pain. Surg Gynecol Obstet 91: 524-526.

13. Hopf HW, Hunt TK, West JM, Blomquist P, Goodson WH, et al. (1997) Wound tissue oxygen tension predicts the risk of wound infection in surgical patients. Arch Surg 132: 997- 1004.

14. Scott NB (2010) Wound infiltration for surgery. Anaesthesia 65 Suppl 1: $67-75$.

15. Liu SS, Richman JM, Thirlby RC, Wu CL (2006) Efficacy of continuous wound catheters delivering local anesthetic for postoperative analgesia: a quantitative and qualitative systematic review of randomized controlled trials. J Am Coll Surg 203: 914- 932.
16. Forastiere E, Sofra M, Giannarelli D, Fabrizi L, Simone G (2008) Effectiveness of continuous wound infusion of $0.5 \%$ ropivacaine by $O n-Q$ pain relief system for postoperative pain management after open nephrectomy. $\mathrm{Br} \mathrm{J}$ Anaesth 101 : 841- 847.

17. Salvemini $S$ (2008) New intraoperative therapeutic approach to control postthoracotomy pain in patients who underwent main resection due to lung neoplasm. Minerva Chir 63: 335- 339.

18. Sidiropoulou T, Buonomo O, Fabbi E, Silvi MB, Kostopanagiotou G, et al. (2008) A prospective comparison of continuous wound infiltration with ropivacaine versus single-injection paravertebral block after modified radical mastectomy. Anesth Analg 106: 997- 1001.

19. Catala E, Casas JI, Unzueta MC, Diaz X, Aliaga L, et al. (1996) Continuous infusion is superior to bolus doses with thoracic paravertebral blocks after thoracotomies. J Cardiothorac Vasc Anesth 10: 586- 588.

20. Perttunen K, Nilsson E, Heinonen J, Hirvisalo EL, Salo JA, et al. (1995) Extradural, paravertebral and intercostal nerve blocks for post-thoracotomy pain. Br J Anaesth 75: 541- 547. 